to minimize the rate of unnecessary LND associated morbidity. Although its use in EC is relatively new, SLN biopsy has been shown to be highly accurate for staging purpose. However, some studies reported on decreased sentinel node detection rate among obese patients. Thus, we sought to determine if SLN technique is reliable with certain factors associated with successful mapping in obese EC patients and whether omitting LND impacts oncologic outcomes.

Methodology A prospective cohort study of obese patients $(\mathrm{BMI}) \geq 35 \mathrm{~kg} / \mathrm{m} 2$ ), diagnosed with endometrial carcinoma between 2007 and 2017, comparing surgical and oncological outcomes of two patients cohorts: LND ( \pm SLN) and SLN. 2year progression-free survival (PFS), overall survival (OS), and disease-specific survival (DSS) were analyzed using life tables, Kaplan-Meier survival curves and log-rank tests.

Results Out of 223 patients with median BMI of $40.6 \mathrm{~kg} / \mathrm{m} 2$, 140 patients underwent LND (with or without SLN) and 83 patients underwent SLN. The median operative time for surgical staging in SLN only group was shorter in 47.5 minutes than for patients in the LND \pm SLN group (190.5 minutes (108-393) vs. 238 minutes (131-440), respectively, $(\mathrm{p}<$ $0.001)$ ), and they had reduced estimated blood loss (EBL) compared to the $\mathrm{LND} \pm \mathrm{SLN}$ group $(30 \mathrm{ml}(0-300)$ vs. $40 \mathrm{ml}$ $(0-800 \mathrm{ml}), \mathrm{P}=0.03)$. At a 24 months follow-up cut-off, $98 \%$ of the patients were alive and $95.5 \%$ were free of disease, without significant differences in OS, DSS and PFS between the two groups $(p=0.7, p=0.8$ and $p=0.4$, respectively). Overall, 171 patients underwent SLN biopsy ( \pm LND) and stratified by the tracer used for mapping (ICG versus blue dye). The ICG injected group had higher successful mapping and bilateral detection rates $(92.8 \%$ vs $71.7 \%, \mathrm{p}<0.001$ and $80.2 \%$ vs $43.3 \%, \mathrm{p}<0.001$, respectfully).

Conclusion Omitting LND from surgical staging where SLN is performed was associated with shorter operative time and minimal bleeding without affecting survival. ICG with NIR fluorescence imaging results in higher detection than with blue dye, indicating that ICG should be the dye of choice in obese endometrial cancer patients.

Disclosures We have no disclosures.

\section{SENTINEL LYMPH NODE MAPPING: THE ANSWER TO THE SURGICAL STAGING DILEMMA IN ELDERLY PATIENTS WITH ENDOMETRIAL CANCER}

${ }^{1}$ Liron Kogan, ${ }^{1}$ Emad Matanes, ${ }^{2}$ Michel Wissing, ${ }^{1}$ Cristina Mitric, ${ }^{1}$ Shannon Salvador ${ }^{1}$ Susie Lau, ${ }^{3}$ Walter Gotlieb. . Mcgill University; Division of Gynecologic Oncology, Jewish General Hospital; ${ }^{2}$ Mcgill University; Division of Cancer Epidemiology, Department of Oncology; ${ }^{3}$ Jewish General Hospital; Mcgill University

\subsection{6/ijgc-2020-ESG0.55}

Introduction/Background Nearly half of women diagnosed with endometrial cancer (EC) are 70 years old or older. Furthermore, elderly patients ( $>65$ years) have been at risk for adverse perioperative outcomes throughout various surgical procedures and have a poorer prognosis which is related to both aggressive disease and under-treatment. We aimed to compare surgical and oncological outcomes between elderly (>65 year-old), intermediate to high risk, endometrial cancer patients undergoing staging with sentinel lymph node (SLN) staging and pelvic lymphadenectomy (LND).

Methodology A prospective cohort study of elderly patients diagnosed with endometrial carcinoma between 2007 and 2017, treated in a single center in Montreal, comparing surgical and oncological outcomes of 3 endometrial cancer patients cohorts in non-overlapping eras, undergoing surgical staging including: LND, LND+SLN or SLN. 2-year progression-free survival (PFS), overall survival (OS), and disease-specific survival (DSS) were analyzed using life tables, KaplanMeier survival curves and log-rank tests.

Results Out of 278 patients with a median age of 73 (65-91) years, that were staged and met the study inclusion criteria, 84 patients underwent LND only, 120 underwent SLN followed by LND and 74 patients had only SLN. Patients in the SLN group had significantly less dissected nodes (mean of 5.4 nodes vs 10.4 and 10.0 in the SLN+LND and the LND cohorts, respectively, $\mathrm{p}<0.001$ ), shorter surgeries with a median time of 199 minutes (range, 75-393) compared to 231 (range, 125-403) and 229 (range, 151-440) minutes in the SLN+LND and LND cohorts, respectively $(\mathrm{p}<0.001)$ and minimal estimated blood loss with a median estimated blood loss of $20 \mathrm{ml} \mathrm{(5-150)}$ vs. $25 \mathrm{ml} \mathrm{(5-800])} \mathrm{and} \mathrm{40(5-400)} \mathrm{in}$ the SLN+LND and LND cohorts, respectively $(\mathrm{p}=0.002)$. $42.4 \%, 19.8 \%$ and $36.2 \%$ of the all cohort received vaginal brachytherapy, external beam radiation and chemotherapy, respectively, with significantly more patients in the SLN group receiving brachytherapy $(54.1 \%$ vs $41.7 \%$ and $33.3 \%$ in the SLN+LND and LND cohorts, respectively, $p=0.03$ ). 2-year overall survival and progression free survival were not significantly different between the 3 groups $(p=0.45, p=0.51$, respectively). On multivariable analysis, adjusted factors that were statistically significant on univariable analysis (age, ASA score, stage, grade, LVSI), adding SLN was associated with better OS, (HR 0.2, CI [0.1-0.6], P=0.006) and PFS (HR 0.5 , CI [0.1-1.0], $\mathrm{P}=0.05$ ).

Conclusion SLN based surgical staging is feasible, precise, affects adjuvant treatment and associated with better surgical and oncological outcomes in intermediate and high risk elderly patients.

Disclosures We have no disclosures.

\section{TUMOUR MOLECULAR FEATURES PREDICT ENDOMETRIAL CANCER PATIENTS' SURVIVAL AFTER DIFFERENT SURGICAL APPROACHES}

Yibo Dai, Jingyuan Wang, Luyang Zhao, Zhiqi Wang, Jianliu Wang. Peking University People's Hospital

\subsection{6/ijgc-2020-ESGO.56}

Background The Cancer Genome Atlas (TCGA) project has shed light on the vital role of tumour molecular features in predicting endometrial cancer (EC) patients' survival. This study aims to investigate the survival impact of surgical approaches on EC patients with different molecular features. Methodology 473 eligible EC patients from TCGA database were selected. Clinicopathological characteristics and genetic features of open and minimally invasive surgery (MIS) group were compared. To analyse the prognostic impact of surgical approach, survival analyses were conducted in patients with different genetic alterations. Finally, a simplified model based on molecular features was established to help select patients suitable for MIS or open surgery.

Results In the eligible patients, 291 (61.5\%) received open surgery and $182(38.5 \%)$ received MIS. Clinicopathological and genetic features were comparable between the two groups except the year of diagnosis $(\mathrm{p}<0.001)$. Among all patients, 4 
genetic features (POLE mutation [POLEmt], microsatelliteinstability high [MSI-H], homologous recombination defect [HRD], MUC16 mutation [MUC16mt]) showed significant overlap. In Kaplan-Meier survival analyses, MIS and open surgery brought similar survival outcome in patients with POLEmt, MSI-H, HRD or MUC16mt. But in POLE wild type, non MSI-H, non HRD, or MUC16 wild type patients, MIS resulted in shorter recurrence-free survival (RFS) $(p=0.008,0.015,0.003,0.008)$. Based on TCGA classification, POLE ultramutated and MSI hypermutated type had similar prognosis after two surgeries, while copy-number low type without CTNNB1 mutation and copy-number high type with TP53 mutation showed more rapid recurrence after MIS $(p=0.048$ and 0.037$)$. Further analyses were done to simplify the model. In patients with $\geq 1$ of the 4 features (POLEmt, MSI-H, HRD or MUC16mt), MIS and open surgery brought comparable overall survival and RFS $(p=0.339$ and 0.969); for patients with none of the features, especially those with wild type CTNNB1 or TP53 mutation, longer RFS was observed in open surgery group $(\mathrm{p}=0.001,<0.001,<0.001$, respectively). All the results of Kaplan-Meier analyses were verified by Cox regressions.

Conclusion The molecular features of EC are related to patients' prognosis after different surgical approaches. MIS should be recommended in patients with POLEmt, MSI-H, HRD or MUC16mt for similar survival outcome and less perioperative complications compared to open surgery.

Disclosures This work was supported by the National Natural Science Foundation of China (81972426, 81202041, 81672571, 81874108 and 81802607), Special Projects for Strengthening Basic Research of Peking University (BMU2018JC005), National Key Technology R\&D Program of China (2019YFC1005200 and 2019YFC1005201).

The authors have no potential conflict of interest to disclose.

\section{THE POSITIVE PELVIC LYMPH NODES IN ENDOMETRIAL CANCER - HISTOPATHOLOGICAL PARAMETERS AS PREDICTORS}

${ }^{1}$ Aljosa Mandic, ${ }^{2}$ Dunja Kokanov, ${ }^{3}$ Bojana Gutic, ${ }^{3}$ Tatjana Ivkovic Kapicl, ${ }^{3}$ Slobodan Maricic, ${ }^{3}$ Nenad Solajic. 'University of Novi Sad; Medical Faculty; Oncology Institute of Vojvodina; ${ }^{2}$ Oncology Institute of Vojvodina; Oncology Institute of Vojvodina; ${ }^{3}$ University of Novi Sad; Medical Faculty; Oncology Institute of Vojvodina

\subsection{6/ijgc-2020-ESG0.57}

Introduction/Background Endometrial cancer is the most common malignancy of the female reproductive tract. Lymph node metastases are an important prognostic factor in endometrial cancer. Several prognostic factors have been shown to correlate with lymph node metastasis: depth of myometrial invasion, cervical infiltration, histologic grade of the tumor, tumor diameter, serous histology, lymphovascular invasion, and positive peritoneal cytology.

Methodology Finding the pathohistological parameters that will indicate with greater certainty the possibility of metastases in the lymph nodes, on the basis of which it will be evaluated whether such patients should undergo lymphadenectomy or not. A retrospective analysis of patients with endometrial cancer who underwent surgery at the Oncology Institute of Vojvodina (Clinic for Operative Oncology - Department of Gynecology) in the period from 2012 to 2018. The study included 120 patients who underwent hysterectomy with bilateral adnexectomy and pelvic lymphadenectomy.

Results Among patients who had lymph node metastases, there were statistically significant more patients $(p<0.01)$ with endometrial cancer of histological type 2, with depth of myometrial invasion greater than 50\%, cervical stroma infiltration, lymphovascular invasion, and positive peritoneal cytology.

Conclusion Histopathological parameters such as type 2 endometrial cancer, myometrial invasion depth greater than 50\%, cervical stroma infiltration, lymphovascular invasion and positive peritoneal cytology increase the chances of lymph node metastases. Tumor size $(>2 \mathrm{~cm})$ as well as histologic grade did not correlate with a higher incidence of lymph node metastases. In this study, both the parametrial infiltration and the number of lymph nodes removed have clinical significance, but not statistical significance.

\section{AN AUDIT OF THE USE OF VAGINAL BRACHYTHERAPY IN ENDOMETRIAL CANCERS}

Bhargavi llangovan, Ganapathy Raman, S Ayyappan. Madras Cancer Care Foundation

\subsection{6/ijgc-2020-ESG0.58}

Introduction/Background Vaginal Brachytherapy is an effective treatment modality to prevent local recurrence in endometrial cancers. We did an audit of the Endometrial cancer cases treated by an oncology group practice over a period of 5 years to assess the use of VBT.

Methodology The data of 106 patients treated for endometrial cancers between 2014 and 2019 by an oncology group across 2 tertiary care centres was retrospectively analysed.

The indications of vaginal brachytherapy, number of fractions, dose per fraction, prescription points, vaginal stenosis on follow up and use of vaginal dilators were the variables collected and were entered in an excel sheet.

Results A total of 106 patient data was entered and analysed. The mean age of the patients was 60 years. The radiotherapy details were available for 84 patients. Of the 84 patients analysed, 59 patients (70\%) received adjuvant Vaginal Brachytherapy, while $25(30 \%)$ did not. Of the patients who received VBT, $32(54.2 \%)$ patients received VBT as a boost after Pelvic RT (figure 1).

VBT BOOST: Among the 32 patients who received vaginal Brachytherapy as a boost after Pelvic RT, only $4(12.5 \%)$ patients had cervix involvement. Lower uterine segment was involved in 12 patients.

The EBRT dose was $45-50 \mathrm{~Gy}$ in 25 to 28 fractions.

Abstract 165 Table 1 The stagewise and grade wise numbers

\begin{tabular}{lc}
\hline STAGE & NUMBER \\
\hline $\mathrm{IA}, \mathrm{G} 1$ & 6 \\
$\mathrm{IA}, \mathrm{G} 2$ & 3 \\
$\mathrm{IA}, \mathrm{G} 3$ & 5 \\
$\mathrm{IB}, \mathrm{G} 1$ & 4 \\
$\mathrm{IB}, \mathrm{G} 2$ & 3 \\
$\mathrm{II}$ & 2 \\
$\mathrm{III} A$ & 2 \\
\hline
\end{tabular}

